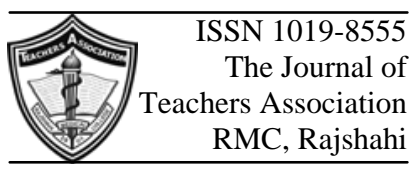

\title{
Editorial
}

\section{GERD and Asthma}

\section{Md. Abdul Ahad*}

It is estimated that more than 75 percent of patients with asthma also experience gastro esophageal reflux disease (GERD). People with asthma are twice as likely to have GERD as those people who do not have asthma. Of those people with asthma, those who have a severe, chronic form that is resistant to treatment are most likely to also have GERD.

GERD is the backward flow of stomach acids into the esophagus. When this acid enters the lower part of the esophagus, it can produce a burning sensation, commonly referred to as heartburn. If left untreated, GERD can eventually lead to lung damage, esophageal ulcers, and in some instances Barrett's esophagus, a condition that can eventually lead to esophageal cancer.

Although studies have shown a relationship between asthma and GERD, the exact relationship is uncertain. GERD may worsen asthma symptoms; however, asthma and some asthma medications may worsen GERD symptoms. On the other hand, treating GERD often helps to also relieve asthma symptoms, further suggesting a relationship between the two conditions.

Doctors most often look at GERD as the cause of asthma when:

- Asthma begins in adulthood

- Asthma symptoms get worse after a meal, after exercise, at night or after lying down

- Asthma doesn't respond to the standard asthma treatments.
As previously mentioned, the exact link between the two conditions is uncertain. However, there are a few possibilities as to why GERD and asthma may coincide. One possibility is that the acid flow causes injury to the lining of the throat, airways and lungs, making inhalation difficult and often causing a persistent cough.

Another possibility for patients with GERD is that when acid enters the esophagus, a nerve reflex is triggered, causing the airways to narrow in order to prevent the acid from entering. This will cause a shortness of breath.

Aside from these possible relationships between asthma and GERD, one study showed there was an increase in the rate of GERD in patients with asthma who were treated with asthma medications known as beta-adrenergic bronchodilators. However, further studies must be done before the relationship between GERD and these drugs can be fully understood.

If a patient has both asthma and GERD, it is important that he consistently take asthma medications as prescribed by the doctor, as well as controlling his exposure to asthma triggers as much as possible.

Fortunately, many of the symptoms of GERD can be treated and/or prevented by taking steps to control or adjust personal behavior. Some of these steps include:

- The head end of the bed should be raised by six inches to allow gravity to help keep the stomach's contents in the stomach. (Piles of 
pillows should not be used because this puts the body into a bent position that actually aggravates the condition by increasing pressure on the abdomen.)

- Meals should be taken at least three to four hours before lying down, and bedtime snacks should be avoided.

- Smaller meals should be taken with moderate portions of food.

- A healthy weight has to be maintained to eliminate unnecessary intra-abdominal pressure caused by extra pounds.

- Consumption of fatty foods, chocolate, peppermint, coffee, tea, colas, and alcohol - all of which relax the lower esophageal sphincter and tomatoes and citrus fruits or juices, which contribute additional acid that can irritate the esophagus should be limited.

- Smoking has to be given up which also relaxes the lower esophageal sphincter.

- Loose belts and clothing should be used.

Aside from these steps, over-the-counter antacids can often relieve GERD symptoms. However, if after one to two weeks these medications do not help to alleviate symptoms, doctor may need to prescribe medications that block or limit the amount of stomach acid. Under rare circumstances, GERD may only be treatable through surgery. 